\title{
THE STRUCTURE OF GRAPHS ON $\boldsymbol{n}$ VERTICES WITH THE DEGREE SUM OF ANY TWO NONADJACENT VERTICES EQUAL TO $n-2$
}

\author{
Do Nhu An ${ }^{a^{*}}$
}

${ }^{a}$ The Faculty of Information Technology, Nha Trang University, Khanh Hoa, Vietnam

*Corresponding author: Email: andn@ntu.edu.vn

\section{Article history}

Received: January $10^{\text {th }}, 2021$

Received in revised form ( $\left.1^{\text {st }}\right)$ : May $19^{\text {th }}, 2021 \mid$ Received in revised form $\left(2^{\text {nd }}\right)$ : July $13^{\text {th }}, 2021$ Accepted: July $16^{\text {th }}, 2021$

Available online: October $4^{\text {th }}, 2021$

\begin{abstract}
Let $G$ be an undirected simple graph on $n$ vertices with the degree sum of any two nonadjacent vertices equal to $n-2$ and let $\alpha(G)$ be the cardinality of a maximum independent set of $G$. We show, for $n \geq 3$ is an odd number then $\alpha(G)=2$ and $G$ is a disconnected graph; for $n \geq 4$ is an even number then $2 \leq \alpha(G) \leq(n+2) / 2$, where if $\alpha(G)=2$ then $G$ is a disconnected graph, otherwise $G$ is a connected graph.
\end{abstract}

Keywords: Connected graph; Disconnected graph; Maximum independent set; Regular graph.

DOI: http://dx.doi.org/10.37569/DalatUniversity.11.4.830(2021)

Article type: (peer-reviewed) Full-length research article

Copyright (C) 2021 The author(s).

Licensing: This article is licensed under a CC BY-NC 4.0 


\section{INTRODUCTION}

The concepts and symbols in this article are referenced from the Handbook of Combinatorics (Graham et al., 1995). Let $G=(V(G), E(G))$ be a simple undirected graph on $n$ vertices, where $V(G)$ is the vertex set and $E(G)$ is the edge set of graph $G$. We use $|V(G)|$ and $|E(G)|$ to denote the number of vertices and edges of $G$. In $G$, the edge of two vertices $u$ and $v$ is denoted by $(u, v)$, the degree of vertex $v$ is denoted by $\operatorname{deg}(v)$, and the minimum degree of the vertices is denoted by $\delta$ or $\delta(G)$. A graph on $n$ vertices is called complete and denoted by $K_{n}$ if its vertices have degree $n-1$. A graph is called a $k$-regular graph if all its vertices have degree $k$. A subset of the vertices in a graph is called independent set if no two vertices in this set are adjacent. A maximum independent set is an independent set that is not a subset of any other independent set. The cardinality of a maximum independent set in $G$ is denoted by $\alpha(G)$. A subset of the vertices in a graph is called a clique if any two of its vertices are adjacent.

The graph $H=(W, F)$ is called a subgraph of $G=(V(G), E(G))$ if $W \subseteq V(G)$ and $F \subseteq E(G)$. Let $v$ be a vertex of $G$; we use $G-v$ to denote the subgraph which is obtained by deleting vertex $v$ and edges attached to $v$ from $G$. Likewise, if $B \subseteq V(G)$, then $G-B$ is a subgraph of $G$ obtained by deleting $B$ from $G$. A graph is connected if any two of its vertices are connected by a path. A component of $G$ is a maximal connected subgraph of $G$. The number of components of $G$ is denoted by $\omega(G)$.

Now, we use the notation $\sigma_{2}(G)=n-2$ to indicate that the graph $G$ on $n$ vertices with the degree sum of any two nonadjacent vertices in $G$ is equal to $n-2$ and $G(n):=\left\{G:|V(G)|=n, \sigma_{2}(G)=n-2\right\}$.

An $(2008,2019)$ has defined the structure of graphs in $G(n)=\{G:|V(G)|=n$, $\left.\sigma_{2}(G)=n-1\right\}$ and proved that recognizing the Hamiltonian graph in $G(n)$ is an easy problem. In this article, we will define the structure of graphs in $G(n)=\{G:|V(G)|=n$, $\left.\sigma_{2}(G)=n-2\right\}$ and show for $n \geq 3$ is an odd number and for every $G \in G(n)$ that $\alpha(G)=2$ and $G$ is a disconnected graph. We also show for $n \geq 4$ is an even number that $2 \leq \alpha(G) \leq(n+2) / 2$ and that $G$ is a disconnected graph if $\alpha(G)=2$. Otherwise, $G$ is a connected graph.

\section{RESULTS}

Let $n \geq 3$ and $G \in G(n)=\left\{G:|V(G)|=n, \sigma_{2}(G)=n-2\right\}$. In $G$, a vertex of degree $n-1$ is called a total vertex, and the set of total vertices in $G$ is denoted by $T(G)$.

For every $G \in G(n)$, we first note by $\sigma_{2}(G)=n-2$ that $G \neq K_{n}$.

Suppose that $u$ and $v$ are any two nonadjacent vertices in $G$. We denote the set of vertices that are not adjacent to $u$ by $N_{u}$ and the set of vertices that are not adjacent to $v$ by $N_{v}$. Then $Z:=V(G) \backslash N_{u} \cup N_{v}$ is a set of vertices that are adjacent to both $v$ and $u$, and $A:=N_{u} \cap N_{v}$ is a set of vertices that are not adjacent to $v$ and $u$. Obviously, $V(G)=Z \cup$ $N_{u} \cup N_{v}$ and $T(G) \subseteq Z$. 
Remark 1. Let $n \geq 3$ and $G \in G(n)$. Then $|Z|=|A|$.

Proof.

For every $u, v \in V(G)$ and $(u, v) \notin E(G)$, we have $|N(u)|=n-1-\operatorname{deg}(u)$, $|N(v)|=n-1-\operatorname{deg}(v)$, and $\operatorname{deg}(u)+\operatorname{deg}(v)=\sigma_{2}(G)=n-2$. By the inclusionexclusion principle, $\quad|Z|=|V(G)|-\left|N_{u} \cup N_{v}\right|=|V(G)|-\left(\left|N_{u}\right|+\left|N_{v}\right|-|A|\right)=$ $n-[n-1-\operatorname{deg}(u)+n-1-\operatorname{deg}(v)-|A|]=|A|$ and therefore $|Z|=|A|$.

We are interested in two cases of the number of vertices of $G$.

\subsection{The case where $n$ is an odd number}

Theorem 1. Let $n \geq 3$ be an odd number and $G \in G(n)$. Then $G$ is a disconnected graph.

\section{Proof.}

First, we prove that in $G$ any two nonadjacent vertices have different degrees.

Indeed, let $u, v$ be two nonadjacent vertices in $G$ and $\operatorname{deg}(u)=\operatorname{deg}(v)$. Then, by $\sigma_{2}(G)=n-2$ and $\operatorname{deg}(u)+\operatorname{deg}(v)=n-2$, it follows that $\operatorname{deg}(u)=\operatorname{deg}(v)$ $=(n-2) / 2$, which is a contradiction with $n$ is an odd number. Therefore, $\operatorname{deg}(u) \neq$ $\operatorname{deg}(v)$.

Next, we will prove that $V(G)=N_{u} \cup N_{v}$ and $N_{u} \cap N_{v}=\emptyset$.

Without loss of generality, we may assume that $\delta=\operatorname{deg}(u)<\operatorname{deg}(v)=$ $n-2-\delta$, where $0 \leq \delta \leq[(n-2) / 2]$. Since $A=N_{u} \cap N_{v}$ is a set of vertices that are both nonadjacent to $u$ and $v$, it follows that $A=\emptyset$. (If not, let $a \in A$ and by $\sigma_{2}(G)=$ $n-2, \operatorname{deg}(u)+\operatorname{deg}(v)=\operatorname{deg}(u)+\operatorname{deg}(a)=\operatorname{deg}(a)+\operatorname{deg}(v)=n-2$. This shows that $\operatorname{deg}(u)=\operatorname{deg}(v)=\operatorname{deg}(a)=(n-2) / 2$, a contradiction with $n$ being an odd number.) By Remark 1 and $A=\emptyset$, we have $Z=\emptyset$ and therefore $V(G)=N_{u} \cup N_{v}$, $N_{u} \cap N_{v}=\varnothing$.

In addition, by (1) and $\sigma_{2}(G)=n-2$, and since vertex $v \in N_{u}$ is not adjacent to the vertices of $N_{v}$ in $G$, it follows that the vertices of $N_{v}$ have degree $\delta$ (similar to the degree of vertex $u$ ) and that these vertices are adjacent in $G$. In other words, the vertices of $N_{v}$ form a clique $K_{\delta+1}$ in $G$. Also, the vertices of $N_{u}$ have degree $n-2-\delta$ (similar to the degree of vertex $v$ ) and the vertices of $N_{u}$ form a clique $K_{n-1-\delta}$ in $G$. And by (2), it follows that $G$ is a disconnected graph and is denoted by $G=K_{\delta+1} \oplus K_{n-1-\delta}$, where $0 \leq \delta \leq[(n-2) / 2]$.

Theorem 1 is proved.

Figure 1 illustrates disconnected graphs corresponding to $\delta=0,1,2$ in $G(7)$. 


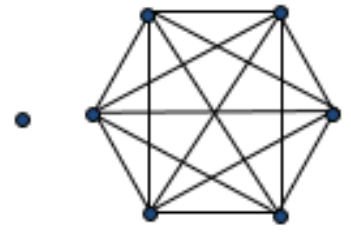

$\delta=0$

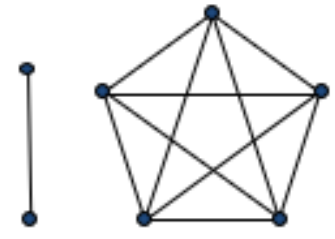

$\delta=1$

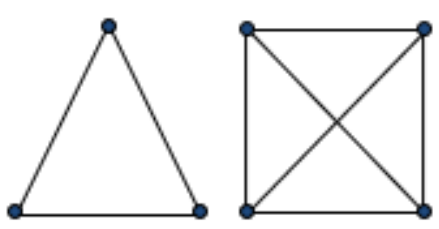

$\delta=2$

Figure 1. Disconnected graphs in $G(7)$

\subsection{The case where $\boldsymbol{n}$ is an even number}

Theorem 2. Let $n \geq 4$ be an even number, $G \in G(n)$, and $S$ is an independent set in $G$. Then

a) if $|S| \geq 3$, the vertices of $S$ have degree $(n-2) / 2$ in $G$.

b) if $G$ is a disconnected graph, $G$ has exactly two components.

Proof.

a) Indeed, let $x, y, z$ be any three nonadjacent vertices of $S$. Then, by $\sigma_{2}(G)=n-2$ and $\operatorname{deg}(x)+\operatorname{deg}(y)=\operatorname{deg}(x)+\operatorname{deg}(z)=\operatorname{deg}(y)+\operatorname{deg}(z)=n-2$, it follows that $\operatorname{deg}(x)=\operatorname{deg}(y)=\operatorname{deg}(z)=(n-2) / 2$. Moreover, because the vertices $x, y, z$ are chosen arbitrarily, we can say that the vertices of $S$ have degree $(n-2) / 2$ in $G$.

b) Suppose that $G$ has more than two components, and $x, y, z$ are three arbitrary vertices such that each vertex belongs to a component of $G$. Then, by Theorem $2 \mathrm{a}$, $\operatorname{deg}(x)=\operatorname{deg}(y)=\operatorname{deg}(z)=(n-2) / 2$. This shows that each component in $G$ has at least $1+(n-2) / 2$ vertices and that the number of vertices in $G$ is $n=|V(G)| \geq$ $3(1+(n-2) / 2)=3 n / 2>n$, a contradiction. Therefore, $G$ has only two components.

Theorem 3. Let $n \geq 4$ be an even number and $G \in G(n)$. Then

(a) $0 \leq|T(G)| \leq \delta \leq(n-2) / 2$.

(b) $2 \leq \alpha(G) \leq(n+2) / 2$.

(c) $\alpha(G)=(n+2) / 2 \Leftrightarrow|T(G)|=(n-2) / 2$.

(d) $\alpha(G)=n / 2 \Rightarrow|T(G)|=0$.

Proof.

a) Clearly, $\delta \leq(n-2) / 2$. Indeed, because if $\delta>(n-2) / 2$, then $n-2=$ $\sigma_{2}(G) \geq 2 \delta>2(n-2) / 2=n-2$, which is a contradiction. Moreover, each total 
vertex must be adjacent to other vertices in $G$, so that the degree of each vertex is not less than $|T(G)|$, i.e., $\delta \geq|T(G)|$. Thus, $0 \leq|T(G)| \leq \delta \leq(n-2) / 2$.

Recall that for $n$ is an even number and $n \bmod 4 \neq 0$, then $\delta=(n-2) / 2$ is an even number and the total vertex has degree $n-1$, an odd number. Therefore, $k=|T(G)|$ must be an even number.

b) Let $S$ be a maximum independent set in $G, \alpha(G)=|S|$.

First, it is clear that by $\sigma_{2}(G)=n-2, G \neq K_{n}$, and therefore $\alpha(G) \geq 2$. Next, we prove that $\alpha(G) \leq(n+2) / 2$. Suppose otherwise, $\alpha(G)>(n+2) / 2$. By $n \geq 4$, $|S|=\alpha(G)>(n+2) / 2 \geq(4+2) / 2=3$. By Theorem $2 \mathrm{a}$, the vertices in $S$ have degree $(n-2) / 2$. Moreover, each vertex of $S$ must be adjacent to $(n-2) / 2$ vertices of $V(G) \backslash S$ in $G$. But this cannot happen because the number of vertices of set $V(G) \backslash S$ is $|V(G) \backslash S|=n-|S|<n-(n+2) / 2=(n-2) / 2$. This contradiction shows that $\alpha(G) \leq(n+2) / 2$.

c) Suppose that $\alpha(G)=(n+2) / 2$ and $S$ is a maximum independent set in $G$. By $n \geq 4,|S|=\alpha(G)=(n+2) / 2 \geq(4+2) / 2=3$, so $|S| \geq 3$, and by Proposition 3a, the vertices of $S$ have degree $(n-2) / 2$ in $G$. Moreover, $|V(G) \backslash S|=n-(n+2) / 2=$ $(n-2) / 2$ and each vertex of $S$ must be adjacent to $(n-2) / 2$ vertices of $V(G) \backslash S$ in $G$ and by $\sigma_{2}(G)=n-2$, all the vertices of $V(G) \backslash S$ are total vertices; thus, we get $T(G)=$ $V(G) \backslash S$ and $|T(G)|=(n-2) / 2$.

Conversely, suppose that $|T(G)|=(n-2) / 2$. We will show that $S:=V(G) \backslash T(G)$ is a maximum independent set in $G$. Obviously, each vertex of $S$ must be adjacent to $(n-2) / 2=|T(G)|$ total vertices in $G$, and by $\sigma_{2}(G)=n-2$, the vertices in $S$ have degree $\delta=(n-2) / 2$ and are nonadjacent in $G$. Therefore, $S$ is an independent set in $G$. But $|S|=|V(G)|-|T(G)|=n-(n-2) / 2=(n+2) / 2$, and by Theorem $3 \mathrm{~b}, S$ is a maximum independent set in $G, \alpha(G)=|V(G) \backslash T(G)|=(n+2) / 2$.

d) Suppose that $S$ is a maximum independent set of $G$ and $|S|=\alpha(G)=n / 2$. We prove that $T(G)=\emptyset$ and so $G$ is a $(n-2) / 2$-regular graph.

First, for $n=4$ it is easy to show by $\alpha(G)=2$ that $G=K_{2} \oplus K_{2}$ is a 1-regular disconnected graph. Now, we consider the case $n \geq 6$. Let $X:=V(G) \backslash S$. By $n \geq 6$, $|S|=|X|=n / 2 \geq 3$. By Theorem $2 \mathrm{a}$, the vertices of $S$ have degree $\delta=(n-2) / 2$, and therefore the vertices of $S$ must be adjacent to $(n-2) / 2$ vertices of $X$ in $G$. Thus, for each vertex $s \in S$, there exists only one vertex $x \in X$ such that $x$ and $s$ are nonadjacent, and $x$ must be adjacent to some other vertices of $S$ in $G$. (If not, $S \cup\{x\}$ is an independent set in $G$, a contradiction for $S$ is a maximum independent set of $G$.) Moreover, by $\operatorname{deg}(x)=(n-2) / 2$, there exists a vertex $y \in X$ that is not adjacent to vertex $x$ in $G$. This shows that $X$ does not contain the total vertex and that $G$ is a $(n-2) / 2$-regular graph.

Theorem 3 is proved. 
Theorem 4. Let $n \geq 6$ be an even number and $G \in G(n)$. Then

a) $\alpha(G)=2$ if and only if $G$ is a disconnected graph.

b) if $3 \leq \alpha(G) \leq(n+2) / 2, G$ is a connected graph, and $G$ contains $k$ total vertices and $n-k$ vertices of degree $\delta=(n-2) / 2$, where $0 \leq k=|T(G)| \leq(n-2) / 2$.

Proof.

Suppose that $\alpha(G)=2$, we will prove that $G$ is a disconnected graph.

Without loss of generality, we can suppose that $S=\{u, v\}$ is a maximum independent set of $G, \operatorname{deg}(u)=\delta$, and $\operatorname{deg}(v)=n-2-\delta$. First, we have $A=\emptyset$ (because if $A \neq \emptyset$ and let $a \in A$, then $\{u, v, a\}$ is an independent set in $G$, which is a contradiction with $\alpha(G)=2$ ). Since $A=\emptyset$ and by Remark $1, Z=\emptyset$ and $T=\emptyset$, and so we get $V(G)=N_{u} \cup N_{v}, N_{u} \cap N_{v}=\emptyset$. Next, by $\alpha(G)=2$, each pair of vertices of $N_{u}$ must be adjacent in $G$. (If not, let $x, y \in N_{u}$ and $(x, y) \notin E(G)$, then $\{x, y, u\}$ is an independent set in $G$, which is a contradiction with $\alpha(G)=2$.) Therefore, the vertices of $N_{u}$ form a clique $K_{\operatorname{deg}(v)+1}=K_{n-1-\delta}$ in $G$. Similarly, each pair of vertices of $N_{v}$ must be adjacent in $G$, and these vertices form a clique $K_{\operatorname{deg}(u)+1}=K_{\delta+1}$ in $G$. In addition, by $\sigma_{2}(G)=n-2$, the vertices of $N_{v}$ are not adjacent to the vertices of $N_{u}$. These results show that $G$ is a disconnected graph and $G=K_{\delta+1} \oplus K_{n-1-\delta}, 0 \leq \delta \leq(n-2) / 2$.

Conversely, let $G$ be a disconnected graph. We will show that $\alpha(G)=2$.

By Theorem 2b, graph $G$ has two components. Let $G=G_{1} \oplus G_{2}$, where $G_{1}$ and $G_{2}$ are connected subgraphs of $G$. We will prove that $G_{1}$ and $G_{2}$ are complete graphs and so $\alpha(G)=2$. Therefore, Theorem $4 \mathrm{a}$ is true.

Indeed, without loss of generality we may assume that $x \in V\left(G_{1}\right), y \in V\left(G_{2}\right)$, and $\operatorname{deg}(x) \leq \operatorname{deg}(y)$. Since the vertices in $G_{1}$ are not adjacent to the vertices in $G_{2}$, and by $\sigma_{2}(G)=n-2$, the vertices in $G_{1}$ must have the same degree as vertex $x$ and the vertices in $G_{2}$ must have the same degree as vertex $y$. Now we consider the following two cases: $\operatorname{deg}(x)=\operatorname{deg}(y)$ and $\operatorname{deg}(x)<\operatorname{deg}(y)$.

For $\operatorname{deg}(x)=\operatorname{deg}(y)$ and by $\operatorname{deg}(x)+\operatorname{deg}(y)=n-2$, we have $\operatorname{deg}(x)=$ $\operatorname{deg}(y)=(n-2) / 2$. Then $\left|V\left(G_{1}\right)\right|=(n-2) / 2+1=n / 2=\left|V\left(G_{2}\right)\right|$. It follows that $G_{1}$ and $G_{2}$ are complete graphs $K_{n / 2}$ and so $G=K_{n / 2} \oplus K_{n / 2}$. For $\operatorname{deg}(x)<\operatorname{deg}(y)$ and since $\sigma_{2}(G)=n-2$, each pair of vertices in $G_{1}$ must be adjacent. In other words, $G_{1}$ is a complete graph $K_{\operatorname{deg}(x)+1}$. Analogously, each pair of vertices in $G_{2}$ must be adjacent and $G_{2}$ is a complete graph $K_{\operatorname{deg}(y)+1}$, therefore $G=K_{\operatorname{deg}(x)+1} \oplus K_{\operatorname{deg}(y)+1}$. In both cases above we get the result that $G_{1}$ and $G_{2}$ are complete graphs.

Note that Theorem $4 \mathrm{a}$ is also true for $n=4$. 
b) Let $S$ be a maximum independent set of $G$. First, since $|S|=\alpha(G) \geq 3$ and by Theorem 2a, all vertices of $S$ have degree $(n-2) / 2$ in $G$. Moreover, by $\sigma_{2}(G)=n-2$, it follows that each vertex of $V(G) \backslash S$ has degree either $(n-1)$ or $(n-2) / 2$ in $G$. In other words, $G$ contains $k$ total vertices and $(n-k)$ vertices of degree $\delta=(n-2) / 2$, where $0 \leq k=|T(G)| \leq(n-2) / 2$ (by Theorem 3a).

Next, in order to show that $G$ is a connected graph, we consider the following two cases: $T(G) \neq \varnothing$ and $T(G)=\varnothing$.

- For $T(G) \neq \emptyset$. Clearly, $G$ is a connected graph because $G$ contains the total vertex.

- $\quad$ For $T(G)=\emptyset$. Then, $G$ is an $\delta-$ regular graph for $\delta=(n-2) / 2$.

Now, suppose otherwise - that $G$ is a disconnected graph. Then, by Theorem $2 \mathrm{~b}$, $G=G_{1} \oplus G_{2}$, where $G_{1}$ and $G_{2}$ are components of $G$. Without loss of generality, we may assume that $\left|V\left(G_{1}\right)\right| \leq n / 2 \leq\left|V\left(G_{2}\right)\right|$. However, the vertices in $G_{1}$ have degree $\delta=$ $(n-2) / 2$, so $\left|V\left(G_{1}\right)\right| \geq \delta+1=(n-2) / 2+1=n / 2$. It follows that $\left|V\left(G_{1}\right)\right|=$ $\left|V\left(G_{2}\right)\right|=n / 2$. Moreover, by $\sigma_{2}(G)=n-2, G_{1}$ and $G_{2}$ must be a complete graph $K_{\delta+1}$, and so $G=K_{\delta+1} \oplus K_{\delta+1}$. It follows that $\alpha(G)=2$, which is a contradiction with the supposition $\alpha(G) \geq 3$. Therefore, $G$ is a connected graph. Theorem $4 \mathrm{~b}$ is proved.
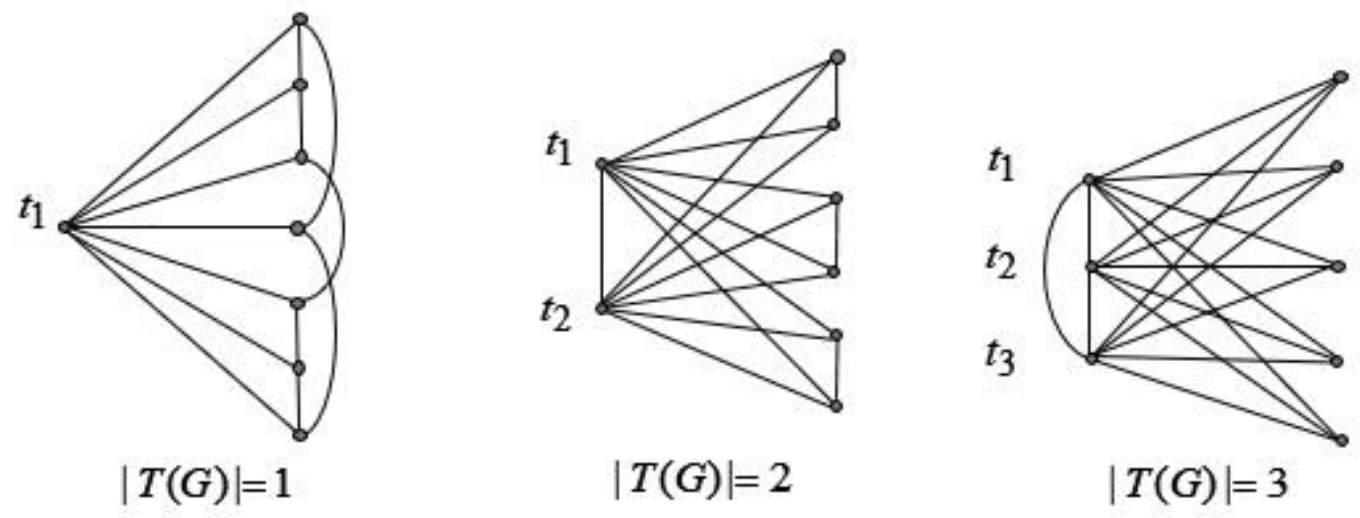

Figure 2. Connected graphs for $|T(G)|=1,2,3$ in $G(8)$
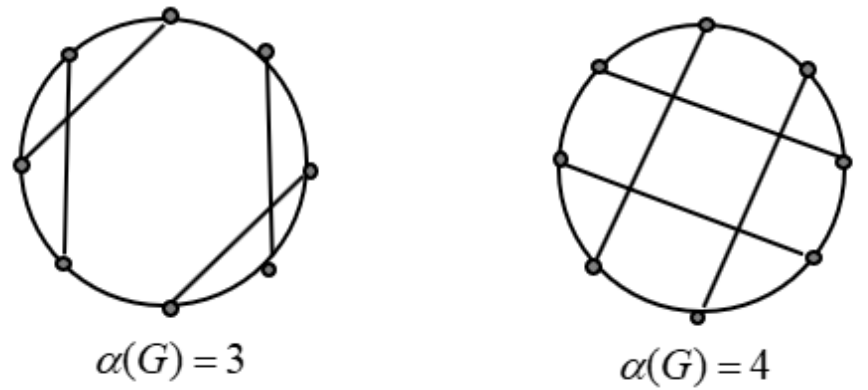

Figure 3. 3-regular graphs in $G(8)$ 
Figure 2 illustrates connected graphs for $\delta=3$ and $|T(G)|=1,2,3$ in $G(8)$, respectively. Figure 3 illustrates 3-regular graphs with $\alpha(G)=3$ and $\alpha(G)=4$ in $G(8)$.

Theorem 4 is proved.

\section{CONCLUSION}

For $G(n)=\left\{G:|V(G)|=n, \sigma_{2}(G)=n-2\right\}$ and $G \in G(n)$, we have shown that if $n \geq 3$ is an odd number, then $G$ is a family of disconnected graphs $K_{\delta+1} \oplus K_{n-1-\delta}$, $\delta=0,1,2, \ldots,[(n-2) / 2]$. For $n \geq 4$ is an even number, there are two cases: If $\alpha(G)=2$, then $G$ is a family of disconnected graphs $K_{\delta+1} \oplus K_{n-1-\delta}, \delta=0,1,2, \ldots,(n-$ $2) / 2$. If $3 \leq \alpha(G) \leq(n+2) / 2$, then $G$ is a family of connected graphs that contains $k$ total vertices and $n-k$ vertices of degree $\delta=(n-2) / 2$, where $0 \leq k \leq(n-2) / 2$. When $k=0, G$ is a $\delta$-regular graph.

\section{REFERENCES}

An, D. N. (2008). Some problems about Hamiltonian cycle in special graphs. The 2nd International Conference on Theories and Applications of Computer Science (ICTACS'09) - Journal of Science and Technology, Vietnam Academy of Science and Technology, 6(5A-Special issue), 57-66.

An, D. N. (2019). Recognizing the Hamiltonian graph on $\mathrm{n}$ vertices with $\sigma_{2}(G)=n-1$ is an easy problem. International Journal of Advanced Research in Computer Science, 10(2), 42-45.

Graham, R. L., Grötschel, M., \& Lovász, L. (1995). Handbook of combinatorics (1st Ed.). North Holland. 